\title{
CFD simulation of sucrose flow field in the stem of greenhouse tomato seedling
}

\author{
J iheng $\mathrm{Ni}^{*}$, J ingtao Dong, I kram Ullah, Hanping Mao \\ (School of Agricultural Engineering, Jiangsu University, Zhenjiang 212013, Jiangsu, China)
}

\begin{abstract}
Sucrose is the main constituent of assimilation transport from source to sink. In order to study the transport mechanism of sucrose, the sucrose at stem was taken as a research object and the sucrose flow field in tomato stem was analyzed using CFD ANSYS FLUENT simulation. The results showed that the sucrose pressure distribution was lower at bottom node and higher at middle node. The velocity of sucrose at different nodes was higher at the bottom node and lower at middle node and assimilation flow direction of the first, second, and third leaf from the bottom was towards the roots. The result of verification experiment showed that sucrose content measured at different nodes was higher at middle node than that of bottom node. Results of this study confirmed that ANASYS FLUENT can better simulate sucrose migration in greenhouse tomato stem. This study provides a new method for studying the partitioning mechanism of tomato assimilates in greenhouse.
\end{abstract} Keywords: greenhouse tomato, sucrose flow field, computational fluent dynamic, rule of distribution DOI: $10.25165 /$ j.ijabe.20221501.6061

Citation: Ni J H, Dong J T, Ullah I, Mao H P. CFD simulation of sucrose flow field in the stem of greenhouse tomato seedling. Int J Agric \& Biol Eng, 2022; 15(1): 111-115.

\section{Introduction}

The crop growth model is a strong tool for greenhouse production and management of greenhouse environment. Dry matter partitioning model of greenhouse tomato is an important part of tomato growth model ${ }^{[1]}$. The distribution of assimilate products to different organs has a decisive effect on the yield and quality of tomato fruits in greenhouse. However, because of poor understanding of partitioning mechanism, dry matter partitioning model was a weak segment and hot topic of crop growth model ${ }^{[2]}$. So, strengthening the study on the distribution mechanism of assimilated products in greenhouse tomatoes and promoting more transportation of assimilated products in fruits will play an important role in improving the production and management of greenhouse tomatoes in China.

Researchers had put forward many hypotheses to explain the mechanism of dry matter partitioning. The source-sink hypothesis was the most widely used method to describe the distribution of dry matter in greenhouse tomatoes ${ }^{[3]}$. But Marcelis thought that there was a shortcoming about the method to express sink or source strength $^{[4]}$. The current research is mainly to clarify the analysis mechanism of assimilates by analyzing the growth of source and sink organs, without considering the effect of flow on the distribution of assimilates. Due to the lack of this part of research, the mechanism of assimilate distribution is not clear.

Plant physicists proved that sucrose is the main form that was transported from source to sink organ ${ }^{[5,6]}$. How to describe the mechanism of assimilates was transported from source organ to

Received date: 2020-08-01 Accepted date: 2021-11-21

Biographies: Jingtao Dong, MS, research interest: greenhouse crop simulation, Email: 1062690252@qq.com; Ikram Ullah, PhD, research interest: greenhouse crop cultivation, Email: ikram2155@gmail.com; Hanping Mao, PhD, Professor, research interest: greenhouse equipment, Email: maohp@ujs.edu.cn.

*Corresponding author: Jiheng Ni, PhD, Associate Professor, research interest: crop growth model and simulation. School of Agricultural Engineering, Jiangsu University, Zhenjiang 212013, Jiangsu, China. Tel: +86-15806106972, Email: nijiheng@163.com. sink organ, Minchin et al. ${ }^{[7]}$ thought that the assimilates flow is defined by the pressure difference between sources and sinks and influenced by the resistance of the phloem pathway and was called Münch hypothesis ${ }^{[8,9]}$. According to the Münch hypothesis, the assimilated content gradually decreased from the source to sink. A number of analytical and numerical models of phloem flow based on the Münch hypothesis have been published, Knoblauch ${ }^{[10]}$ used micro-fluid to test Münch hypothesis. Mammeri and Sellier ${ }^{[11]}$ found a surface model of nonlinear, non-steady state phloem transport and a steady state model of phloem flow. In the study of Savage et al. ${ }^{[12]}$, the velocity of phloem transport varied over time of cucumber seedling. The research work has clarified aspects of phloem loading and phloem ultra-structure on phloem transport. But what remains unknown is whether phloem transport is stable over space and time and how the structure of phloem network impact carbon movement in plants ${ }^{[13]}$. So, the objective of this research is to study the dynamic change of phloem flow over space and time.

Computational fluid dynamic (CFD) is a flow field method that combines modern fluid mechanics, numerical mathematics, and computer science ${ }^{[14]}$. It can efficiently solve various complex flow calculation problems through discrete mathematical methods. And now, it has been widely used in ecology, medicine and plant physiology research. FLUENT is a popular commercial CFD software package in the world. It contains various fields of flow simulation software, which can quickly and accurately get the flow field in the pipeline $e^{[15]}$.

The seedling stage is an important period of tomato growth cycle. Studies proved that the differentiation of leaves and flowers of tomato has ended at the seedling stage ${ }^{[16]}$. Therefore, the quality of seedling growth has an important impact on the yield and quality of tomatoes and tomato plants can provide a simple sink and source model of phloem transport ${ }^{[17]}$. Because there is no temperature change when assimilates was transported from source to sink. So, it can be looked at the transportation of assimilates as a steady-state process. The assimilates are in the form of sucrose solution at transported tissue, so it can be regarded 
as an impressible fluid. The movement and transportation process is calculated and analyzed by establishing a three-dimensional flow simulation model in ANSYS FLUENT software. In view of this, we take the stem as the main pipe, the position of the leaf as the input, and the sucrose flow as the internal fluid to construct a three-dimensional pipe simulation model to simulate and analyze the flow process of sucrose in the stem, to explain the distribution mechanism of assimilates in greenhouse tomato seedlings.

\section{Materials and methods}

\subsection{Model description}

The software FLUENT was used to study the distribution of sucrose and its daily change at stem. There are four steps to process. First, a geometric model of tomato stem was constructed. Second, girding of the geometric model was done based on the size of tomato stem. Third, the FLUEN soft parameters were determined according to the experiment result. Fourth, input parameters and run FLUENT.

\subsection{Control equation}

The model of sucrose flow field at tomato stem was found based on these assumptions as follows: 1) Sucrose flow at phloem was defined impressible Newton fluid and the flow stage was turbulent; 2) The temperature was evenly distributed at tomato stem, and the transport process was steady; 3) Energy dissipation due to viscosity in the sieve wall was ignored. The transportation of sucrose was according to the mass conservation and energy conservation equation. So, the equation of described sucrose flow at stem was as follows:

$$
\frac{\partial}{\partial t}(\rho \varphi)+\operatorname{div}(\rho \overrightarrow{\mathrm{u}} \varphi)=\operatorname{div}\left(\Gamma_{\varphi} \operatorname{grad} \varphi\right)+S_{\varphi}
$$

where, $\varphi$ is general transmission; $t$ means time, $\mathrm{s} ; \rho$ is the fluid density, $\mathrm{kg} / \mathrm{m}^{3} ; \overrightarrow{\mathrm{u}}$ is the velocity of fluid, $\mathrm{m} / \mathrm{s} ; \Gamma_{\varphi}$ is diffusion coefficient of $\varphi ; S_{\varphi}$ is source term.

\subsection{Gridding of tomato stem}

In this study, tomato stem was chosen as the research object (Figure 1), the initial stage was as follows: the mean node length of tomato stem was $0.73 \mathrm{~cm}$ and the diameter of input port was considered the diameter of the leaf petiole. The height of the pipe model was mean plant height which was taken as $22.7 \mathrm{~cm}$. The difference between input port and output port height was $0.5 \mathrm{~cm}$.

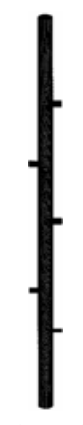

Figure 1 Grading of greenhouse tomato stem under seedling stage

The unstructured tetrahedral mesh was used to describe the computational stem in greenhouse tomato seedling stage. In order to ensure the accuracy of grid, the maximum thickness of the whole grid was set to $0.001 \mathrm{~m}$, and the grid parameters need to be adjusted continuously during the process of meshing. The quality of the grid was controlled according to EquiAngle Skew standard. The meshed structure is shown in Figure 1.

\subsection{Selection of turbulence model}

The turbulence models are divided into standard turbulence models and RNG turbulence models. Compared to the standard turbulence model, RNG-turbulence model has the same format and the predicted results are more precise because of adding items to the equation.

\subsection{Model selection}

\subsubsection{Experiment design}

An experiment was conducted in a $12 \mathrm{~m} \times 18 \mathrm{~m}$ greenhouse compartment, which is part of the multi-span Venlo type greenhouse of the department of agricultural equipment and engineering (Jiangsu University, Jiangsu Province, China) from February to July 2017.

In this experiment, indeterminate growing tomato cultivar "Fenhong906" was used. Seeds of tomato were sown in trays filled with a commercial peat. About $30 \mathrm{~d}$ after sowing, seedlings were transplanted into cultural pots which were filled with perlite and placed on cultural bed. The used pot was $20 \mathrm{~cm}$ in height, the diameter of pot at the top was $32 \mathrm{~cm}$ and the diameter of the bottom pot was $26 \mathrm{~cm}$. The plant density was $2.5 \mathrm{~m}^{-2}$. Plant nutrient and chemical pest and disease control were conducted according to commercial standards. Plants were irrigated by Hoagland nutrient solution. When tomatoes grew under seedling stage, two group samples were randomly selected at 8:00, 10:00, 12:00, 14:00, and 16:00 of the day, and plant transportation tissue was decomposed into nodes and leaf petioles.

The sucrose content of every node, leaf petiole near to leaf and leaf petiole near to stem were measured ${ }^{[17]}$ and was divided into two groups, one group was used to model found and the other was used to validate the model.

\subsubsection{Parameters of material defined}

In this study, sucrose flow was defined as flow material of FLUENT. The density and viscosity of sucrose flow were defined. Data of density and viscosity of sucrose flow ${ }^{[19]}$.

\subsubsection{Boundary conditions setting}

According to the trait of sucrose transport of tomato, the boundary conditions of phloem flow were divided into velocity boundary condition and pressure boundary condition. The velocity boundary was velocity of sucrose flow at leaf petiole, which was from experiment data. The velocity of sucrose at leaf petiole was calculated by Hagen-Poiseuille equation.

$$
V_{i}=\frac{\pi r^{4}}{8 \eta} \frac{\Delta P}{L}
$$

where, $V$ is the velocity of sucrose at leaf petiole, $\mathrm{m} / \mathrm{s} ; r$ is the diameter of leaf petiole; $L$ is the length of leaf petiole, $\mathrm{cm} ; i$ is leaf sequence; $\eta$ is the sap viscosity, $\mathrm{kg} / \mathrm{m}^{3[19]}$.

According to Gould' result, the driving force for the transportation of sucrose in Vascular bundle mainly comes from the osmotic potential, which is calculated according to van't Hoff equation.

$$
\Delta P=\left(C_{1 i}-C_{2 i}\right) R T / 342
$$

where, $C_{1}$ and $C_{2}$ are sucrose content at leaf petiole near leaf and near stem, respectively, $\mathrm{mg} / \mathrm{g} ; R$ is gas constant and got the value 0.083 ; $T$ is Kelvin temperature, $\mathrm{K}$ (in this research, $T=293.15 \mathrm{~K}$ ); 342 means sucrose Molecular Weight.

In the pressure boundary condition, according to tomato growth mechanism, the press of growth point was set 0 , and the press of root was set at $0.001 \mathrm{~Pa}$.

Effect of turbulence must be considered and was calculated by following equations ${ }^{[18]}$ :

$$
I=0.16 R_{\mathrm{e}}^{(-1 / 8)}
$$




$$
\operatorname{Re}=\frac{v d \rho}{\eta}
$$

where, $I$ is the turbulence intensity; Re is Reynolds number; $v$ is the velocity of sucrose flow, $\mathrm{m} / \mathrm{s} ; d$ is the measured diameter of leaf petioles, $\mathrm{m}$.

Input the sucrose velocity of each leaf petiole into the model, the model can predict the sucrose pressure and velocity at every node, so the distribution of the sucrose at stem can be got. Used other independent data to validate the distribution of sucrose at stem.

\section{Results}

\subsection{Pressure distribution cloud map at nodes under tomato seedling stage}

In order to get pressure distribution simulation, the velocity of sucrose flow of each leaf petiole at 8:00, 10:00, 12:00, 14:00,16:00 was used as input into FLUENT and run the software. It resulted in pressure distribution cloud (Figure 2). From Figure 2, we can see that the press of sucrose flow at stem was lowest at 8:00, then gradually increased, and reached its maximum value at 10:00 followed by a decrease at 12:00 and then again increased at 14:00 followed by a decrease at 16:00. Sucrose flow in tomato stem had two peaks at a day, one peak was at 10:00, the other was at 14:00. The distribution of sucrose flow pressure under different nodes showed the same change at different times.

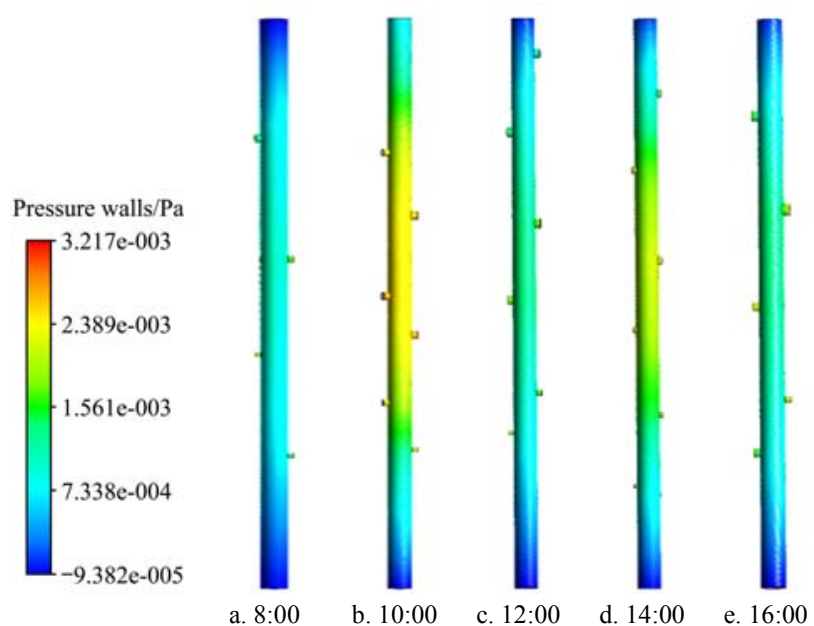

Note: each column at every map cloud means leaf petiole

Figure 2 Diurnal change of sucrose pressure cloud under stem of greenhouse tomato at different time

Pressure of sucrose flow in the middle stem was higher than that of upper growth point and lower part of stem near to roots. The overall pressure value of sucrose flow was not more than $0.03 \mathrm{~Pa}$. In our results, the maximum pressure of sucrose flow at phloem predicted by FLUNT was $0.03 \mathrm{~Pa}$, far less than those as found in previously conducted studies. In Knoblauch results, the pressure of sucrose flow at source was 1.21 MPa and Hall got the maximum pressure was $4.00 \mathrm{MPa}$ at leaf petiole ${ }^{[10]}$. The reason was that in Knoblauch and Hall study, the pressure data was measured at leaf petiole but according to our results, the pressure of sucrose flow was predicted at stem. Mammeri and Sieler ${ }^{[11]}$ found a surface to simulate the sucrose flow at stem of Matua Ngahere tree. According to his results, the pressure data of sucrose flow at stem was $0.05 \mathrm{~Pa}$, which was closed to our results.

The diurnal change of sucrose pressure in stem was the same as photosynthesis rate change of tomato leaf, which proved that there were two net photosynthetic rate high points on the same day, and photosynthesis rate had high correlation with sucrose content. At 8:00, the photosynthesis rate of tomato leaf was low, so assimilates produced by leaf were not much. With the time-lapse, the photosynthetic rate of tomato leaf gradually increased, and more and more assimilation products were produced. At 10:00, the photosynthetic rate of greenhouse tomato reached maximum value, and the rate of assimilation products reached the highest. More and more assimilates are transported from leaf to transported tissues of stem in the form of sucrose, which resulted in the maximum pressure in the transported tissues. The photosynthesis rate of tomato leaf was decreased from 10:00 to 12:00, so tomato leaf produced less assimilates and the pressure of sucrose at stem transported tissue was decreased too. At 14:00, photosynthesis rate of tomato leaf reached the next peak, so the pressure of sucrose at stem was increased.

The sucrose pressure in the stem decreased gradually from the central to both ends. The central of sucrose pressure was at third, fourth, fifth node when plant grew four (Figure 2a), five (Figure 2e), and six (Figures 2b, 2c, and 2d) leaves, respectively. This difference in sucrose flow distribution could be resulted due to two possible reasons. Firstly, the capacity of leaf photosynthesis is significantly affected by leaf position and leaf age. Leaf photosynthetic capacity of newly growing leaves is less than that of mature leaves. In the seedling stage, upper leaves were growing leaf, the photosynthesis rate was less than that of middle leaf, so assimilates produced by middle leaf were more than that of upper leaf. Secondly, in seedling stage, the main organs consuming assimilate were roots and upper growth points. When assimilate were transported to roots and growth points, sucrose was consumed. Therefore, the pressure of sucrose at both ends of stems is the lowest, and the pressure of assimilation products at the middle of stems is the highest.

\subsection{Velocity distribution cloud map at nodes under tomato seedling stage}

Input the velocity values of sucrose flow of each leaf petiole at 8:00, 10:00, 12:00, 14:00, 16:00 into FLUENT and run the soft. The simulated velocity distribution cloud of sucrose at stem is shown in Figure 3. From Figure 3, it can be seen that the change of sucrose velocity distribution at stem was the same as the pressure distribution during a day, with peaks at 10:00 and 14:00 respectively. At 8:00, the velocity of sucrose flow was lowest, with time-lapse, the velocity of sucrose flow gradually increased and reached maximum value at 10:00, then decreased gradually from 10:00 to 12:00, after 12:00, the velocity of sucrose flow at stem increased gradually and reach next peak at 14:00. The maximum velocity of sucrose flow was $119 \mu \mathrm{m} / \mathrm{s}$, slightly less than that of previously studied ${ }^{[14]}$, which was measured by carbon-11 labeled photo-assimilates and got the data was $119 \mu \mathrm{m} / \mathrm{s}$.

The distribution of sucrose flow velocity at different nodes was the complete opposite of pressure at different nodes. At the central stem of plant, the velocity of sucrose flow was lowest, and increased gradually from central to both ends.

Many scientists proved that assimilates were transported from source to sink organ, and the force came from the content difference between sink and source organ. In the experiment, the capacity of photosynthesis rate at middle leaves was bigger than that of other leaves, so assimilates produced by middle leaves were more than those of other leaves. In seedling stage, growing point and root were dominant organs to consume assimilates. When tomato plant grew, assimilates which at growing point and root was consumed quickly and this resulted in the content difference 
between growing point, root and plant central, so the velocity of sucrose flow was increased gradually from plant central to both ends. From Figure 3, it can be seen that assimilates produced by different leaves were transported different organs. In the experiment, assimilates produced by below three leaves were transported to root, and assimilates produced by other leaves were transported to growing point.

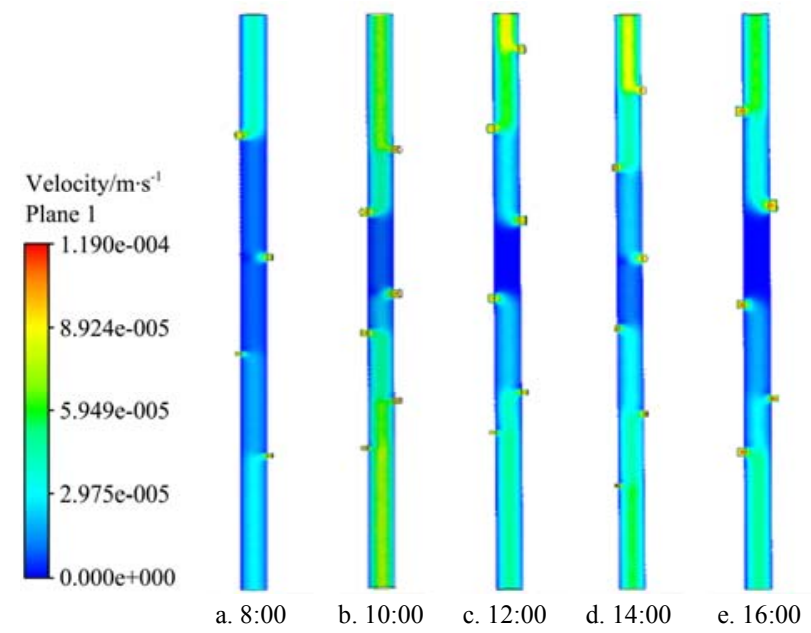

Note: each column at every map cloud means leaf petiole.

Figure 3 Diurnal change of sucrose velocity cloud under stem of greenhouse tomato at different time

\subsection{Model validation}

Using Equations (2) and (3), the velocity of sucrose flow of each leaf petiole at 8:00, 10:00, 12:00, 14:00,16:00 was calculated and then put into FLUENT and run the software. The pressure field in the stem of tomato seedlings was obtained. Using the pressure of different nodes and Equation (3), the sucrose contents of different nodes were obtained, and then compared with the measured sucrose content of each node. The results showed that the predicted sucrose contents of different nodes at different times were in good agreement with the experimental data (RMSE $=0.12 \mathrm{mg} / \mathrm{g}, R^{2}=0.95$ ). Therefore, Fluent soft is a strong tool to study the mechanism of dry matter partitioning.

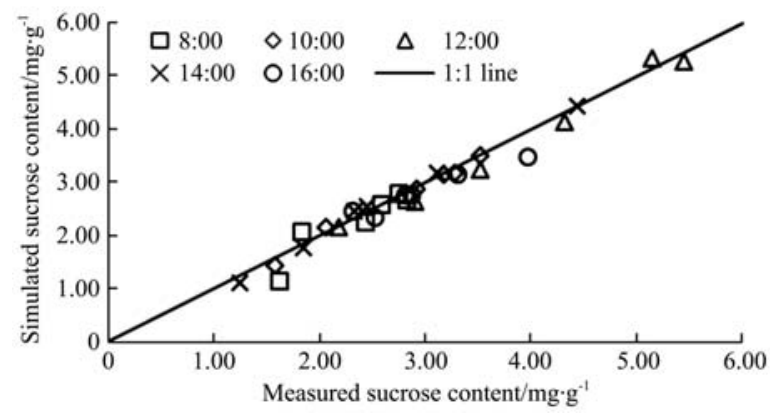

Figure 4 Diurnal change of sucrose content at different nodes of tomato seedling

\section{Discussion}

\subsection{Methods of study long-distance phloem}

Phloem transport is the process by which assimilates produced by photosynthesis in the leaves get distributed within a plant ${ }^{[20]}$. The current hypothesis for phloem transport was Münch hypothesis. According to Münch hypothesis, assimilates were transported through the phloem and the drive force was pressure difference between source and sink. The Münch hypothesis has gained wide acceptance based to a large extent on its simplicity and plausibility, rather than on experimental evidence. In order to prove Münch hypothesis, a number of scientists found phloem mathematics model to test long-distance phloem transport. For example, Thompson and Holbrook ${ }^{[21]}$ found a non-steady-state phloem model, Mammeri and Sellier ${ }^{[11]}$ found a nonlinear non-steady-state phloem model and Cabrita et al. ${ }^{[22]}$ found steady-state phloem model. But these models had too many parameters which was not easily measured. Many scientists used experiment to prove Münch hypothesis. For example, Babst et al. ${ }^{[23]}$ used radioisotopes, Mullendore et al. ${ }^{[24]}$ used magnetic resonance imaging, Savage et al. ${ }^{[12]}$ used dye tracking. But these experiments can measure the velocity of sucrose flow, cannot measure the pressure of sucrose flow. Therefore, it is an urgent need to find a simple method to the found phloem model to predict velocity and pressure of sucrose flow. In our results, phloem is considered as a tiny pipe, and sucrose flow is considered as a fluid flowing in the pipe. The distribution of pressure and velocity field of sucrose flow in phloem was simulated by FLUENT software which simulates the distribution of fluid in the pipe. Compared with measured sucrose content at different nodes, the model can give satisfactory velocity and pressure at different nodes.

Compared with previous studies, using FLUENT to study long-distance phloem transport not only overcomes the shortcomings of too many parameters and difficulties to obtain by using mathematical model methods, but also overcomes the shortcomings of using experimental methods to measure only the flow rate of assimilated products but not the pressure. The fluent method has the advantage of less accurate parameters, so it can be used as a new method to study long-distance transportation of phloem.

\subsection{Relationship between sucrose flow at phloem and source-sink}

A number of early studies have noted that phloem transport was sensitive to changes in plant water status ${ }^{[26]}$. The change of tomato water status was high-low-high from pre-draw to midday under seedling stage ${ }^{[27-29]}$. In our result, the velocity of sucrose at stem was increasing from 8:00 to 10:00, and decreased from 12:00 to $16: 00$. The sucrose flow velocity at stem was also governed by the sink strength. When the sources were strong enough to meet the demand, the velocity of sucrose flow was constant. Another study was conducted to study the day and night time phloem flow velocity of four plant which resulted in no significant difference between day and night phloem flow velocity in tobacco, castor bean and tomato ${ }^{[26]}$. Savage et al. $^{[12]}$ used a novel dye-tracing technique to measure phloem transport velocity in seedling cucumber, he thought that seedling plant appeared carbon limited and reported that the velocity of phloem was determined by source-sink relations. These results coincided with the results of this study. In our results, tomato plant was under source-limit stage, the velocity of phloem was changed.

\section{Conclusions}

The pressure and velocity of assimilates flow are critical to plant establishment and have important implications for assimilates distributions in their natural environments. In this study, the diurnal and spatial changes of sucrose flow in the stem of greenhouse tomato seedlings were simulated. It was verified by measured data and achieved satisfactory prediction results. According to our results, the sucrose transport at phloem coincided with the theory of fluid. So, it was a good choice to use FLUENT soft to study assimilate transport.

However, the result does not consider water relations within 
stem and does not take into account nutrition supplements. One possibility for future work is to study the effect of different electrical conductivity on assimilates flow pressure and velocity, as this would improve our understanding of assimilates distribution and its interaction with water and nutrition supplements.

\section{Acknowledgements}

This research was funded by the senior personnel of Jiangsu University foundation (Grant No. 13JDG31) and a project funded by the priority academic program development of Jiangsu Higher Education Institutions (Grant No. 2014037).

\section{[References]}

[1] Heuvelink E. Dry matter partitioning in tomato: validation of a dynamic simulation model. Annals of botany, 1996; 77(1): 71-80.

[2] Patil S B, Mansur C P, Gaur P M, Salakinkop S R, Alagundagi S C. Planting density affected dry matter production, partitioning, and yield in machine harvestable chickpea genotypes in the irrigated ecosystem. International Journal of Plant Production, 2021; 15(1): 29-43.

[3] Marcelis L, Heuvelink E, Goudriaan J. Modelling biomass production and yield of horticultural crops: A review. Scientia Horticulturae, 1998; 74(1-2): 83-111.

[4] Marcelis L F M. Sink strength as a determinant of dry matter partitioning in the whole plant. Journal of Experimental Botany, 1996; 47: 1281-1291.

[5] Farrar J, Pollock C, Gallagher J. Sucrose and the integration of metabolism in vascular plants. Plant Science, 2000; 154(1): 1-11.

[6] Liesche J, Martens H J, Schulz A. Symplasmic transport and phloem loading in gymnosperm leaves. Protoplasma, 2011; 248(1): 181-190.

[7] Minchin P, Thorpe M, Farrar J, Koroleva O. Source-sink coupling in young barley plants and control of phloem loading. Journal of experimental botany, 2002; 53(374): 1671-1676.

[8] Gould N, Morrison D, Clearwater M J, Ong S, Boldingh H, Minchin P E. Elucidating the sugar import pathway into developing kiwifruit berries (Actinidia deliciosa). New Zealand Journal of Crop and Horticultural Science, 2013; 41(4): 189-206.

[9] Thompson M V. Phloem: the long and the short of it. Trends in plant science, 2006; 11(1): 26-32.

[10] Knoblauch M, Peters W S. Münch, morphology, microfluidics-our structural problem with the phloem. Plant, cell \& environment, 2010; 33(9): 1439-1452.

[11] Mammeri Y, Sellier D. A surface model of nonlinear, non-steady-state phloem transport. Mathematical Biosicences and Engineering: MBE, 2017; 14(4): 1055-1069.

[12] Savage J A, Zwieniecki M A, Holbrook N M. Phloem transport velocity varies over time and among vascular bundles during early cucumber seedling development. Plant Physiology, 2013; 163(3): 1409-1418.

[13] Fan W W, Yuan L J, Li Y L. CFD simulation of flow pattern in a bubble column reactor for forming aerobic granules and its development.
Environmental Technology, 2019: 40(27): 3652-3667.

[14] Bendevis M A, Sun Y, Rosenqvist E, Shabala S, Liu F, Jacobsen S-E. Photoperiodic effects on short-pulse $14 \mathrm{C}$ assimilation and overall carbon and nitrogen allocation patterns in contrasting quinoa cultivars. Environmental and Experimental Botany, 2014; 104: 9-15.

[15] Bhaskar K U, Murthy Y R, Raju M R, Tiwari S, Srivastava J, Ramakrishnan N. CFD simulation and experimental validation studies on hydrocyclone. Minerals Engineering, 2007; 20(1): 60-71.

[16] Jensen K H, Lee J, Bohr T, Bruus H, Holbrook N M, Zwieniecki M A. Optimality of the Münch mechanism for translocation of sugars in plants. Journal of the Royal Society Interface, 2011; 8(61): 1155-1165.

[17] Li W, Na C, Zhao X C, Fan H Y, Li T. Accumulation of carbohydrate and regulation of 14-3-3 protein on sucrose phosphate synthase (SPS) activity in two tomato species. Journal of Integrative Agriculture, 2014; 13(2): 358-364.

[18] Westerwalbesloh C, Grünberger A, Stute B, Weber S, Wiechert W, Kohlheyer D, et al. Modeling and CFD simulation of nutrient distribution in picoliter bioreactors for bacterial growth studies on single-cell level. Lab on a Chip, 2015 ; 15(21): 4177-4186.

[19] Honig P. Principles of sugar technology. Elsevier, 2013;773p.

[20] Hall A, Minchin P. A closed - form solution for steady - state coupled phloem/xylem flow using the $\mathrm{L}$ ambert $-\mathrm{W}$ function. Plant, Cell \& Environment, 2013; 36(12): 2150-2162.

[21] De Schepper V, De Swaef T, Bauweraerts I, Steppe K. Phloem transport: a review of mechanisms and controls. Journal of experimental botany, 2013; 64(16): 4839-4850.

[22] Thompson M V, Holbrook N M. Application of a single-solute non-steady-state phloem model to the study of long-distance assimilate transport. Journal of Theoretical Biology, 2003; 220(4): 419-455.

[23] Cabrita P, Thorpe M, Huber G J. Hydrodynamics of steady state phloem transport with radial leakage of solute. Frontiers in Plant Science, 2013; 4 531. doi: 10.3389/fpls.2013.00531.

[24] Babst B A, Ferrieri R A, Gray D W, Lerdau M, Schlyer D J, Schueller M, et al. Jasmonic acid induces rapid changes in carbon transport and partitioning in Populus. New Phytologist, 2005; 167(1): 63-72.

[25] Mullendore D L, Windt C W, Van As H, Knoblauch M. Sieve tube geometry in relation to phloem flow. The Plant Cell, 2010; 22(3): 579-593.

[26] Windt C W, Vergeldt F J, De Jager P A, Van As H. MRI of long - distance water transport: a comparison of the phloem and xylem flow characteristics and dynamics in poplar, castor bean, tomato and tobacco. Plant, Cell \& Environment, 2006; 29(9): 1715-1729.

[27] McCall D, Atherton J. Interactions between diurnal temperature fluctuations and salinity on expansion growth and water status of young tomato plants. Annals of applied biology, 1995; 127(1): 191-200.

[28] Wang Y, Pang Y L, Chen K, Zhai L Y, Shen C C, Wang S, et al. Genetic bases of source-, sink-, and yield-related traits revealed by genome-wide association study in Xian rice. The Crop Journal, 2020; 8(1): 123-135.

[29] Ma Y T, Chen Y J, Zhu J Y, Meng L, Guo Y, Li B G, et al. Coupling individual kernel-filling processes with source-sink interactions into GREENLAB-Maize. Global Change Biology, 2018; 121(5): 961-973. 